\title{
Contribuição de um programa de práticas corporais lúdicas na prevençã̃o de doenças cardiovasculares
}

\author{
Albertina Bonetti \\ Universidade Federal de Santa Catarina \\ a.bonetti@ufsc.br \\ Daiane Wommer \\ Universidade Federal de Santa Catarina \\ daiawom@yahoo.com.br
}

\author{
Geny Aparecida Cantos \\ Universidade Federal de Santa Catarina \\ geny@ccs.ufsc.br \\ Thuani Antunes Corrêa \\ Universidade Federal de Santa Catarina \\ thuani@hotmail.com
}

\section{Resumo}

O objetivo deste trabalho foi relatar como um programa de práticas corporais, denominado Vivências Lúdicas do Coração (VLC), contribuiu para melhorar o estilo de vida de um grupo pertencente a uma comunidade universitária. Este projeto foi desenvolvido em parceria com uma equipe multidisciplinar e multiprofissional que tem trabalhado com prevenção de doenças cardiovasculares, sendo essa mais uma possibilidade de prevenção e reabilitação. No ano de 2011, participaram desse programa 22 pessoas, e os encontros foram realizados três vezes por semana, com duração de uma hora. A metodologia foi centrada no prazer, no lúdico, possibilitando às pessoas realizarem movimentos, estimulando o desenvolvimento da criatividade. Ao longo do ano foram realizadas brincadeiras e pequenas gincanas com balões, bambolês, cordas, bolas, bastões e outros materiais. Essas brincadeiras melhoraram a agilidade, o equilíbrio e a coordenação motora dos participantes. Também foram incluídos variados exercícios localizados e alongamentos, os quais foram muito importantes para desenvolver uma boa flexibilidade. Algumas vezes foram realizadas caminhadas, o que ajudou na melhora do condicionamento físico. Conclui-se que esse programa ocupou um espaço terapêutico de grande importância, abrindo um campo rico de oportunidades para o tratamento e recuperação dos seus integrantes, pois as pessoas criaram melhores condições físicas, mentais e sociais, e desfrutaram de uma melhor qualidade de vida.

Palavras-chave: Prevenção. Atividade física. Vivência.

\section{Contribution of a body practices recreational in the prevention of cardiovascular disease}

\section{Abstract}

The objective of this study was to report as a physical activity program called Experiences recreational heart has contributed to improving the lifestyle of a group belonging to a community college. This project was developed in partnership with a multidisciplinary and multiprofessional, who has worked on prevention of cardiovascular diseases, and this is one more possibility of prevention and rehabilitation. In 2011, 22 people participated in this program, and these meetings were held three times a week, lasting one hour. The methodology was focused on pleasure in play allowing people performing movements, stimulating the 


\section{Extensio $\mid$ Relato de Experiência}

development of creativity. Throughout the year were held games and small competitions with balloons, hula hoops, ropes, balls, sticks and other materials. These games have improved agility, balance and motor coordination of the participants, varied weight training and stretching exercises were also included and these were very important to develop a good flexibility. Sometimes walks were held, which helped in improving fitness. It is concluded that this program occupied a very important therapeutic area, opening a rich field of opportunity for treatment and recovery of its members, because people create better physical, mental and social, and enjoyed a better quality of life.

\section{Keywords: Prevention. Physical activity. Experiencing.}

\section{INTRODUÇÃO}

As doenças cardiovasculares (DCV) são as principais causas de morte no Brasil. Um terço dessas mortes ocorre precocemente em adultos de idade entre 35 e 64 anos. No entanto, se houvesse assistência ou prevenção, poderia ocorrer a diminuição dessas mortes (ISHITANI et al., 2006). Diversos estudos demonstraram que é possível reduzir a carga dessa doença por meio de estratégias preventivas (LEITE et al., 2008; PITANGA et al., 2010).

$\mathrm{Na}$ Universidade Federal de Santa Catarina, observou-se que era grande o número de funcionários, alunos e professores que buscavam atendimento cardiológico. Assim, em setembro de 1997, um grupo de profissionais interessou-se em atuar na prevenção e no controle da doença aterosclerótica na população universitária, criando, então, o Núcleo Interdisciplinar de Pesquisa, Ensino e Assistência à Dislipidemia do Hospital Universitário da Universidade Federal de Santa Catarina (NIPEAD), que inicialmente disponibilizava atendimento cardiológico e nutricional (CANTOS et al., 2007). Posteriormente, os indivíduos passaram também a receber orientações por meio de palestras mensais, as quais propiciaram um espaço para integração, trocas de experiências entre os pacientes, esclarecimentos de dúvidas referentes ao tema abordado, configurando assim um trabalho interdisciplinar. Os temas abordados nas palestras foram baseados nas necessidades indicadas pela própria comunidade e desenvolvidos pela equipe do NIPEAD (SCHUTZ, et al, 2009).

A proposta do NIPEAD foi crescendo e passou a envolver outras atividades, tais como: a Biodanza (CANTOS et al., 2005), uma modalidade aquática (Watsu', Halliwick e Biodanza aquática) (MORRIS, 1994; CUNHA, 1998) e atendimento psicológico: as vivências lúdicas do coração (BONETTI, 2006). A biodanza trabalhou, em grupo, exercícios específicos que estimularam os potenciais genéticos de cada participante. Na verdade, esse processo ocorreu concomitantemente com o outro, de forma que essa comunicação melhorou a autoestima das pessoas e consequentemente o seu bem-estar biológico e psicológico (CANTOS et al., 2007). O atendimento psicológico permitiu-lhes desenvolverem a percepção de si mesmas, promovendo o autoconhecimento, e serviu também para verificar a presença de sinais indicadores de alterações psicológicas (ex.: ansiedade, estresse, somatização, etc.) e desenvolver procedimentos para ajudar a encontrar estratégias de enfrentamento e de resolução de problemas adequadas para lidar com as situações geradoras do adoecimento físico e psicológico. O Watsu (water shiatsu), técnica de massagem japonesa adaptada à água, utilizou uma variedade de alongamentos e movimentos, de forma que o indivíduo pudesse relaxar o corpo e a mente, permitindo o alívio da dor e do estresse. O método Halliwick - técnica de natação desenvolvida por James Mcmillan, em 1949, que associou o seu conhecimento sobre fluidos mecânicos somado a conceitos teóricos e observações realizadas com as reações do corpo humano no ambiente aquático - foi utilizado terapeuticamente de forma que o indivíduo pudesse obter a máxima independência na água (autoconfiança) (CANTOS et al., 2008).

No cenário de medidas preventivas em todos os grupos, a alimentação saudável ocupou lugar de destaque. Para isso, almoços comunitários mensais em todos os grupos foram realizados. O cardápio era prepa- 
rado pelo grupo e analisado por uma nutricionista, que verificava se ele estava balanceado. Os cuidados na sua elaboração e na sua avaliação periódica foram ferramentas importantes na busca da promoção da saúde.

O objetivo deste artigo é relatar como o programa de Vivências Lúdicas do Coração contribuiu para promover mudanças positivas no estilo de vida das pessoas que participaram de um programa de prevenção contra doenças cardiovasculares, dando ênfase às práticas realizadas durante o ano 2011.

\section{MATERIAIS E MÉTODOS}

Trata-se de um relato de experiência (POLIT et al., 2004), cujos dados para análise neste estudo tiveram como fonte as atividades desenvolvidas pelo NIPEAD durante o ano 2011.

Em 2011 foram consultados 712 pacientes no setor de cardiologia. Nessas consultas foi feito um “check-up" personalizado. Após a avaliação clínica, a fim de que se pudesse avaliar o perfil lipídico e o risco cardiovascular, as pessoas foram encaminhadas para realizarem exames laboratoriais no Laboratório de Análises Clínicas do Hospital Universitário (HU) da Universidade Federal de Santa Catarina (UFSC). Após esclarecimento sobre os objetivos do estudo, os métodos e os benefícios, todos os participantes assinaram o termo de consentimento de adesão ao estudo.

O perfil lipídico foi obtido considerando os seguintes exames: Colesterol total (CT), HDL-colesterol (HDL-c), LDL-colesterol (LDL-c) triglicerídeos (TG). Outros exames normalmente solicitados foram: a glicose (Gli), para saber se a pessoa era diabética; ureia e creatinina (para avaliar a função renal); hemograma completo (para investigar anemias e outras alterações sanguíneas; T3, T4 e TSH (para avaliar a função tireoidiana); as enzimas Aspartato Aminotransferase e Alanina Aminotransferase (indicadoras sensíveis de lesão ou proliferação celular, sobretudo hepática). A dosagem de hemoglobina glicada (HbA1C) foi utilizada para o controle glicêmico de pacientes diabéticos. O sódio, potássio, magnésio, cálcio e cloretos constituíram-se em uma investigação secundária, sendo os três últimos marcadores de diestresse; a Microalbuminúria era solicitada muitas vezes aos pacientes diabéticos e hipertensos, por ser um marcador prognóstico de risco cardiovascular e/ou renal.

Para saber o risco cardiovascular de cada paciente, utilizou-se o escore de Framingham, o qual considera idade, sexo, pressão arterial, os valores numéricos dos exames solicitados, tabagismo e diabetes mellitus (DM), classificando a pessoa em baixo, médio e alto risco coronariano em pessoas sem diagnóstico prévio de aterosclerose prévia. Assim, nas consultas de retorno, com base nos resultados laboratoriais obtidos, a cardiologista do referido Núcleo analisava os dados e orientava as pessoas sobre os riscos cardiovasculares.

Depois de passar pelo setor de cardiologia, e receber ou não tratamento medicamentoso, todos os pacientes foram convidados para uma consulta nutricional, mas somente 73 decidiram participar desse tipo de tratamento. O atendimento foi realizado na Unidade de Nutrição (SASC) do HU-UFSC, e as intervenções nutricionais foram inteiramente conduzidas por uma nutricionista desse Núcleo. Na consulta inicial foi aplicado um questionário (ficha nutricional), onde foram coletadas informações gerais, história clínica, história alimentar e dados antropométricos, IMC (Índice de Massa Corporal), GET (Gasto Energético Total) e GEB (Gasto Energético Basal) do paciente. Também foi entregue um exemplo de cardápio, no qual o paciente deveria anotar por quatro dias sua ingestão alimentar. No retorno, ele era orientado a adotar uma alimentação equilibrada e balanceada, enfatizando a promoção de hábitos alimentares saudáveis, mudanças no estilo de vida como, por exemplo, a prática regular de exercícios.

Com a finalidade de orientar os pacientes de forma integrada e divertida, foram organizados almoços comunitários, nos quais os participantes do programa juntamente com a equipe do NIPEAD se reuniam na casa de algum colega, levando um alimento saudável para partilhar com o grupo. Conversando e trocando receitas, eles foram orientados a prepararem seus alimentos de forma saudável e saborosa, sob as óticas higiênico-sanitária, nutricional e sensorial. Outra forma encontrada para orientar os integrantes do projeto de maneira divertida foram as visitas ao supermercado, nas quais os participantes do projeto foram acom- 


\section{Extensio $\mid$ Relato de Experiência}

panhados por uma nutricionista.

Foram realizadas também palestras mensais no HU para todos integrantes do NIPEAD, abrindo espaço para a comunidade em geral. Os assuntos foram relacionados com a prevenção contra as doenças cardiovasculares. Essas reuniões foram conduzidas por diversos palestrantes, que sempre abriam espaço para uma conversa, com o intuito de os ouvintes esclarecerem suas dúvidas.

Entre os vários programas direcionados à promoção da saúde e prevenção de doenças cardiovasculares, foram realizadas várias atividades: Biodanza ${ }^{\circledR}$, Watsu ${ }^{\circledR} /$ Halliwick, atendimento psicológico e Vivências Lúdicas do Coração. Essas atividades foram oferecidas primeiramente para os integrantes do NIPEAD, e posteriormente foi aberto espaço para a comunidade local participar. A inscrição para cada modalidade se deu no início de cada semestre do ano 2011, ou seja, em março e agosto. A divulgação foi realizada via contato direto, em palestras realizadas mensalmente, em consultas cardiológicas e nutricionais e, posteriormente, pelo site da UFSC.

A Biodanza , que proporcionou vivências integradoras para 30 pessoas, foi realizada uma vez por semana, em uma sala de ginástica do Centro de Desportos da UFSC, em sessões que tiveram duração de 2 horas. O atendimento psicológico grupal ocorreu no Serviço de Atenção Psicológica (SAPSI) da UFSC, no Departamento de Psicologia, sendo realizados trinta e três encontros semanais que atenderam cinco mulheres e três homens. A terapia aquática (Watsu'/Halliwick) foi realizada uma vez por semana, em piscina aquecida, durante duas horas, atendendo 20 pessoas. As Vivências Lúdicas do Coração foram realizadas três vezes por semana, com vinte e quatro pessoas na faixa etária de 47 a 67 anos, sendo desenvolvidas nas segundas, quartas e sextas-feiras, no período das 16 horas às 17 horas, na sala de ginástica do Centro de Desportos da UFSC.

Neste último programa, o trabalho foi desenvolvido com a dimensão corporal, ampliando o domínio da experiência e dando estímulos para que a pessoa pudesse ter aspectos de vida mais saudáveis. Nas vivências eram realizadas, de forma a construir o direito ao jogo, ao lúdico e à criatividade, possibilitando, assim, às pessoas sentirem prazer na relação delas com o próprio corpo e com o movimento do outro, por meio de atividades que buscavam a cultura do movimento como a ginástica, as danças, a caminhada, a yoga, o jogo, entre outras práticas. As possibilidades oferecidas pelas práticas corporais de tocar, sentir, massagear e perceber o seu corpo e o do outro foram conduzidas de forma que a pessoa pudesse ter: conscientização do movimento, sensibilidade, autoconhecimento, bem-estar e relaxamento. Em todas as práticas, o lúdico esteve sempre presente. No final de cada encontro os participantes davam depoimentos a respeito das vivências que haviam participado. Estes depoimentos foram registrados em um diário de campo, no qual foram analisadas as manifestações verbais das pessoas, as impressões e os sentimentos que surgiram durante a experiência.

\section{RESULTADOS E ANÁLISE}

Participar de práticas corporais regularmente tem sido aconselhado por diferentes profissionais da área da saúde, a fim de preservar o bem-estar físico, psíquico e social, assim como prevenir, controlar e curar doenças. Recentemente nossa sociedade vem preocupando-se intensamente com a qualidade de vida, e pode-se perceber que o lazer encontra-se como um aspecto bastante importante (PYLRO et al., 2005).

Neste trabalho, o lúdico foi utilizado como forma de lazer, uma maneira de fugir da rotina, da correria e do estresse do dia a dia. Podemos entender como lúdico o jogo, que compreende histórias, dramatizações, canções, danças e outras manifestações artísticas; jogo exclui qualquer atividade profissional, com interesse e tensão. De uma forma geral, entende-se como jogo, toda atividade prazerosa, descomprometida com a realidade, com objetivos característicos e próprios que são atingidos e se encerram com ela. (DOHME, 2003). 
A diferença é que nesse [programa] a gente trabalha o corpo, a mente, aqui estamos trabalhando mais o todo. [...] esta aula é mais comunicativa, e a outra era mais exercícios mesmo, era chão, era pesinho, aqui não, a gente se diverte, dança. (Letícia)

As práticas corporais regulares têm sido propostas para pessoas com predisposição a desenvolver doenças crônicas, como mais uma possibilidade de ajuda (BONETTI, 2006). Nesse sentido, o programa de VLC representou um espaço criativo, misturando prazer, alegria, liberdade, relaxamento, etc. Neste programa, pode-se reconhecer as pluralidades e as heterogeneidades de cada um como um ato indispensável. Além disso, ainda como um ato indispensável, buscou-se o diálogo:

Eu me sinto feliz, alegre. Aqui parece que eu me realizo. Sinto-me contente, converso, sinto-me muito bem mesmo. Eu sempre fui uma pessoa fechada, aqui a gente conversa, a gente ri com todo mundo. Às vezes eu chego em casa e falo que hoje eu me diverti com aquele pessoal da ginástica, foi tão bom. As pessoas estão mais sorridentes, estão mais animadas para tudo. (Gioconda)

Ao longo do ano, foram realizadas brincadeiras e pequenas gincanas com balões, bambolês, cordas, bolas, bastões e outros materiais. Essas brincadeiras trabalharam com a agilidade, o equilíbrio e a coordenação motora dos participantes; exercitar essas habilidades melhora a capacidade motora. Todos os movimentos que as pessoas fizeram envolveram coordenação psicomotora ou neuromuscular. Percebeu-se que, quanto melhor foi a qualidade da coordenação, mais fácil e preciso foi a realização do movimento, o que fez com que o gasto energético fosse menor e o consumo de oxigênio controlado de acordo com a necessidade muscular, diminuindo o nível de fadiga (HOLLMANN apud SILVA, 1998), conforme ilustra este depoimento: "Está melhorando (o corpo), está melhorando. Antes eu não podia mexer muito com as pernas, não podia fazer muita coisa, assim, subir escadas, agora eu estou mais leve." (Lúcia)

$\mathrm{Na}$ ginástica, os pacientes tiveram aulas de bodypump e step. O body pump é um programa de treinamento com sobrecarga, exercícios coreografados e repetitivos que ajuda a melhorar a resistência muscular, o condicionamento físico, e auxilia na perda de gordura e no aumento da massa corporal magra (PFITZINGER et al., 2003). O step, por sua vez, caracteriza-se por movimento básico de subir e descer uma plataforma, de mesmo nome, sincronizado com um compasso musical (WIECZOREK et al., 1997). Essas aulas eram sempre muito divertidas e visivelmente apreciadas pelos participantes. "Na convivência com o pessoal, a gente ri muito. O mais interessante é que ninguém está fazendo pouco do outro. Todo mundo está levando isto na brincadeira, rindo, se divertindo, é uma diversão.” (Carlos)

Realizou-se, ao longo do ano, variados exercícios localizados. Utilizaram-se bastões, halteres e caneleiras, trabalhando diferentes músculos. Cada participante decidia o peso que podia usar, para que não houvesse riscos, e sim benefícios. Alongamentos também foram incluídos e estes foram muito importantes para desenvolver uma boa flexibilidade - essencial na execução de outros exercícios para que não houvesse risco de lesão. Cada pessoa pôde manter o seu nível de flexibilidade de acordo com a estrutura dos componentes (ligamentos, articulações, músculos e outras estruturas envolvidas) do seu aparelho locomotor para que pudesse cumprir as exigências que a prática da respectiva atividade exerceu (DANTAS, 2005).

Outra prática realizada foi o pilates, técnica de conscientização corporal que visou ao fortalecimento dos músculos (SOUZA, 2012). Foram trabalhadas a flexibilidade, a respiração, a força muscular e a postura, utilizando-se o peso do próprio corpo para a execução dos exercícios.

Algumas vezes foram realizadas caminhadas. A caminhada, assim como a corrida, a natação e outras atividades que envolvam exercitar diversos músculos, geraram um ajuste cardiovascular, resultando no aumento da capacidade de se exercitar, na resistência e na força muscular esquelética (MONTEIRO et al., 2007). Essa é uma atividade física simples de ser executada e torna-se mais lúdica quando trabalhada em grupo. 
Eu sinto que saio daqui mais prazeroso, mais disposto [...] que isto também me mantém com um peso para não engordar. Também esta atividade que eu faço aqui me ajudou muito no caminho [no mês de abril a maio realizou a caminhada de Santiago de Compostela], esta minha disposição, isto teve muita influência daqui, porque, se eu não fizesse estes alongamentos, se eu não me mexesse um pouco, acho que o caminho seria mais difícil pra mim. Uma das coisas com que me surpreendi foi a minha superação física, entendes? Ter conseguido fazer $766 \mathrm{~km}$, nunca usei ônibus, nunca usei táxi, fiz tudo a pé. Então, esta atividade aqui me ajudou bastante. (Bernardo)

Todas essas práticas foram realizadas visando à saúde e ao bem-estar dos integrantes do projeto e sempre buscando agradar a todos, de forma que o próprio grupo possuía a liberdade de conversar entre si e com a professora sobre as atividades que lhe faziam bem. Cada integrante pôde realizar as atividades de acordo com sua capacidade e, quando necessário, com a ajuda dos outros participantes, aprendendo a trabalhar e conviver em grupo.

Quando a gente entra em um grupo, de início a gente fica meio introvertido, meio tímido, mas, à medida do convívio, a gente deslancha mesmo e, hoje, a gente se sente muito bem. [...] Este trabalho deste curso melhorou bastante. A gente se sente mais íntimo das pessoas, é com certeza muito gostoso, muito bom. Este convívio com outras pessoas só melhorou. (João Carlos)

\section{CONSIDERAÇÕES FINAIS}

O acesso à orientação nutricional e cardiológico foi fundamental no tratamento e prevenção de doenças cardiovasculares, pois possibilitou o diagnóstico, a identificação precoce e a abordagem adequada dos fatores de risco para doenças cardíacas.

As práticas corporais associadas a dietas equilibradas foram importantes recursos não medicamentosos na terapia e prevenção das doenças cardiovasculares. O programa VLC possuiu uma grande importância terapêutica, pois proporcionou a prática regular e adequada de exercícios físicos, ajudando na recuperação de seus participantes. No cenário de medidas preventivas, este programa ocupou lugar de destaque, contribuindo para um viver mais saudável das pessoas. Muitos movimentos corporais foram ressignificados de forma prazerosa. As pessoas puderam também compartilhar experiências diversificadas em grupo, o que estimulou a participação e a adesão delas ao trabalho interdisciplinar e multiprofissional.

Por outro lado, o trabalho desenvolvido por essa equipe de profissionais permitiu uma prática voltada para um entendimento maior da pessoa, permitindo-a conviver melhor com a situação da doença. As pessoas tiveram maior motivação para adotar hábitos de vida mais saudáveis e puderam refletir sobre o autocuidado e autorresponsabilidade e desfrutaram de uma melhor qualidade de vida. 


\section{REFERÊNCIAS}

BONETTI, A. O Coração e o Lúdico: Vivências corporais para um viver mais saudável de pessoas com doenças aterosclerótica coronariana. Tese de doutorado, Centro de Desportos, UFSC, Florianópolis, dezembro. 2006.

CANTOS GA.; SCHÜTZ R.; ROCHA ME. Associação das técnicas de Watsu e Halliwick com a Biodanza aquática, como forma de melhorar o estresse psicológico de pacientes com doenças crônicas. Revista Pensamento Biocêntrico. v 9, p 69-83, 2008.

CANTOS GA.; SILVA CM.; HERMES EM.; BALÉN MG.; SCHUTZ R. Atendimento multiprofissional e interdisciplinar para pacientes com dislipidemia de uma comunidade universitária: relatos de uma experiência de 10 anos. Revista Ciências da Saúde. v 26, n 2, p 33-8, 2007.

CANTOS GA.; WALTRICK E.; HERMES EM. et.al. Biodanza como nova abordagem terapêutica para pacientes com problemas cardiovasculares. Revista Pensamento Biocêntrico, v 2, p 5-10, 2005.

CUNHA, MCB.; LABRONINI, RHDD.; OLIVEIRA, ASB. \& GABBAI, AA. Hidroterapia. Revista Neurociências, v 6, n 3, p 126-130, 1998.

DANTAS, EHM. Alongamento e flexionamento. 5 ed. Rio de Janeiro: Shape, 2005.

DOHME, V. Atividades lúdicas na educação: o caminho de tijolos amarelos do aprendizado. Petrópolis, RJ: Vozes, 2003.

ERDMANN, A., NASCIMENTO, K. do, SILVA, G da, RAMOS, S. Cuidado de enfermagem e educação em saúde com profissionais do surf. Cogitare Enfermagem, América do Norte, 2007.

ISHITANI, LH.; FRANCO, G. da C.; PERPETUO, IHO e FRANCA, E. Desigualdade social e mortalidade precoce por doenças cardiovasculares no Brasil. Revista de Saúde Pública, v 40, n 4, p 684-691, 2006.

LEITE, ST. et al. Respostas cardiovasculares a mudança postural e capacidade aeróbia em homens e mulheres de meia-idade antes e após treinamento físico aeróbio. Revista Brasileira de Fisioterapia, v 12, n 5, p 392-400, 2008.

MATSUDO, SM.; MATSUDO, VKR. Prescrição e benefícios da atividade física na terceira idade. $\underline{\mathbf{R e}-}$ vista Brasileira de Ciência e Movimento, v 6, n 4, p 19-30, 1992.

MONTEIRO, HL.; ROLIM LMC.; SQUINCA DA. et al. Efetividade de um programa de exercícios no condicionamento físico, perfil metabólico e pressão arterial de pacientes hipertensos. Revista Brasileira de Medicina do Esporte, v 13, p 110, 2007.

MORRIS, DM. Aquatic rehabilitation for the treatment of neurological disorders. Journal of Back and Musculoeskeletal Rehabilitation, v 4, p 297-308, 1994.

PFITZINGER, P.; LYTHE, J. O consumo aeróbico e o gasto energético durante o Bodypump. Fitness \& Performance Journal, v 2, n 2, p 113-121, 2003. 
PITANGA, CPS. et al. Atividade física como fator de proteção para comorbidades cardiovasculares em mulheres obesas. Revista Brasileira de Cineantropometria e Desempenho Humano. v 12, n 5, p 324$330,2010$.

POLIT, DF; BECK CT.; HUNGLER BP. Fundamentos de pesquisa em enfermagem: métodos, avaliação e utilização. Trad. Ana Thorell. 5ª ed. Porto Alegre (RS): Artes Médicas, 2004.

PYLRO, SC.; ROSSETTI, CB. Atividades lúdicas, gênero e vida adulta. Psico-USF, v 10, n 1, p 77-86, 2005.

SCHUTZ, R.; GAZZOLA, J.; BALEN, MGW.; HERMES, EM.; SILVA, CM.; CANTOS, GA. Orientação nutricional na prevenção e controle de doenças cardiovasculares em uma comunidade universitária. Revista Eletrônica Extensio, UFSC, v 6 e 7, 2009.

SILVA, JC. O treinamento e avaliação da coordenação em indivíduos da terceira idade. Monografia da Faculdade de Educação Física, Universidade Estadual Paulista, Rio Claro, 1998.

SOUZA, TM. O Método Pilates Solo na Educação Física: Alguns Benefícios. Monografia, Universidade Federal de Santa Catarina, 2012.

WIECZOREK, SA.; DUARTE, M.; AMADIO, AC. Estudo da força reação do solo no movimento básico de "step". Revista Paulista de Educação Física, v 11, n 2, p 103-15, 1997. 